\title{
The use of non-selective beta-blockers in patients with cirrhosis: more doubts than certainties
}

\author{
Barbara Lattanzi, Vincenza Di Gregorio, Daria D’Ambrosio, Manuela Merli \\ Division of Gastroenterology, Department of Clinical Medicine, Sapienza University of Rome, Rome, Italy \\ Correspondence to: Prof. Manuela Merli. Division of Gastroenterology, Department of Clinical Medicine, Sapienza University of Rome, Rome, Italy. \\ Email: Manuela.merli@uniroma1.it. \\ Provenance: This is a Guest Editorial commissioned by Associate Editor-in-Chief Xingshun Qi (Department of Gastroenterology, General Hospital \\ of Shenyang Military Area, Shenyang, China). \\ Comment on: Kim SG, Larson JJ, Lee JS, et al. Beneficial and harmful effects of nonselective beta blockade on acute kidney injury in liver transplant \\ candidates. Liver Transpl 2017;23:733-40.
}

Received: 11 July 2017; Accepted: 03 August 2017; Published: 24 August 2017.

doi: $10.21037 / \mathrm{amj} .2017 .08 .08$

View this article at: http://dx.doi.org/10.21037/amj.2017.08.08

Since 1980s non-selective beta-blockers (NSBBs) have become the cornerstone of medical treatment for portal hypertension in cirrhotic patients (1). Yet, the evidence for their efficacy to prevent variceal bleeding is derived from prospective trials which largely excluded patients with refractory ascites and renal failure that are patients in which the use of beta-blockers is nowadays under debate (2). In 2010, a prospective observational study, from the same group that had previously supported the use of NSBBs therapy in cirrhosis, suggested that these drugs might increase mortality in patients with refractory ascites. The authors investigated 151 cirrhotic patients with refractory ascites, $51 \%$ were taking propranolol for esophageal varices (it is not specified whether for primary or secondary prophylaxis), while $49 \%$ were not under propranolol treatment. They reported a median survival of 5 months in patients on propranolol versus 20 months in those not receiving this drug and gave a warning about the use of NSBBs in cirrhotic patients with refractory ascites (3). The study was, however, criticized both due to the high mean dosage of NSBBs and due to some relevant differences between the two groups of patients which might have influenced the results. To further investigate their hypothesis, the same authors performed a small crossover study aimed at evaluating the effect of NSBBs on the development of paracentesis-induced circulatory dysfunction (PICD). In this study, ten cirrhotic patients with refractory ascites taking NSBBs were enrolled and monitored before, immediately after and 1 week later after a large volume paracentesis. NSBBs were then discontinued (following endoscopic variceal treatment) and a further paracentesis was repeated followed by the same clinical evaluations. The incidence of PICD decreased from $80 \%$ to $10 \%$, suggesting that NSBBs could have a potentially deleterious effect through further compromising the already impaired hemodynamic balance in patients with advanced cirrhosis and ascites (4). Additional studies were published in recent years (Table 1). Galbois and coworkers, in a retrospective analysis on 68 cirrhotic patients admitted to ICU with sepsis (31 with refractory ascites), reported no difference in mortality between patients receiving or not receiving NSBBs (5). Similarly, Robins and coworkers reported, in a retrospective study with 114 patients with ascites undergoing regular paracentesis, no significant difference in survival between patients using propranolol (mean total daily dose of 40-80 mg daily) and controls (6). Comparable results were reported in 61 cirrhotic patients, in need of paracentesis twice yearly or more frequently in spite of diuretic treatment, with no difference in mortality between patients assuming or not assuming NSBBs (7). Leithead and coworkers also performed a single-centre retrospective study on 322 patients with ascites waiting for liver transplantation and found that NSBBs were not detrimental but associated with a lower mortality. Even in the subgroup of patients with refractory ascites $(n=117)$, NSBB remained independently associated with a lower 


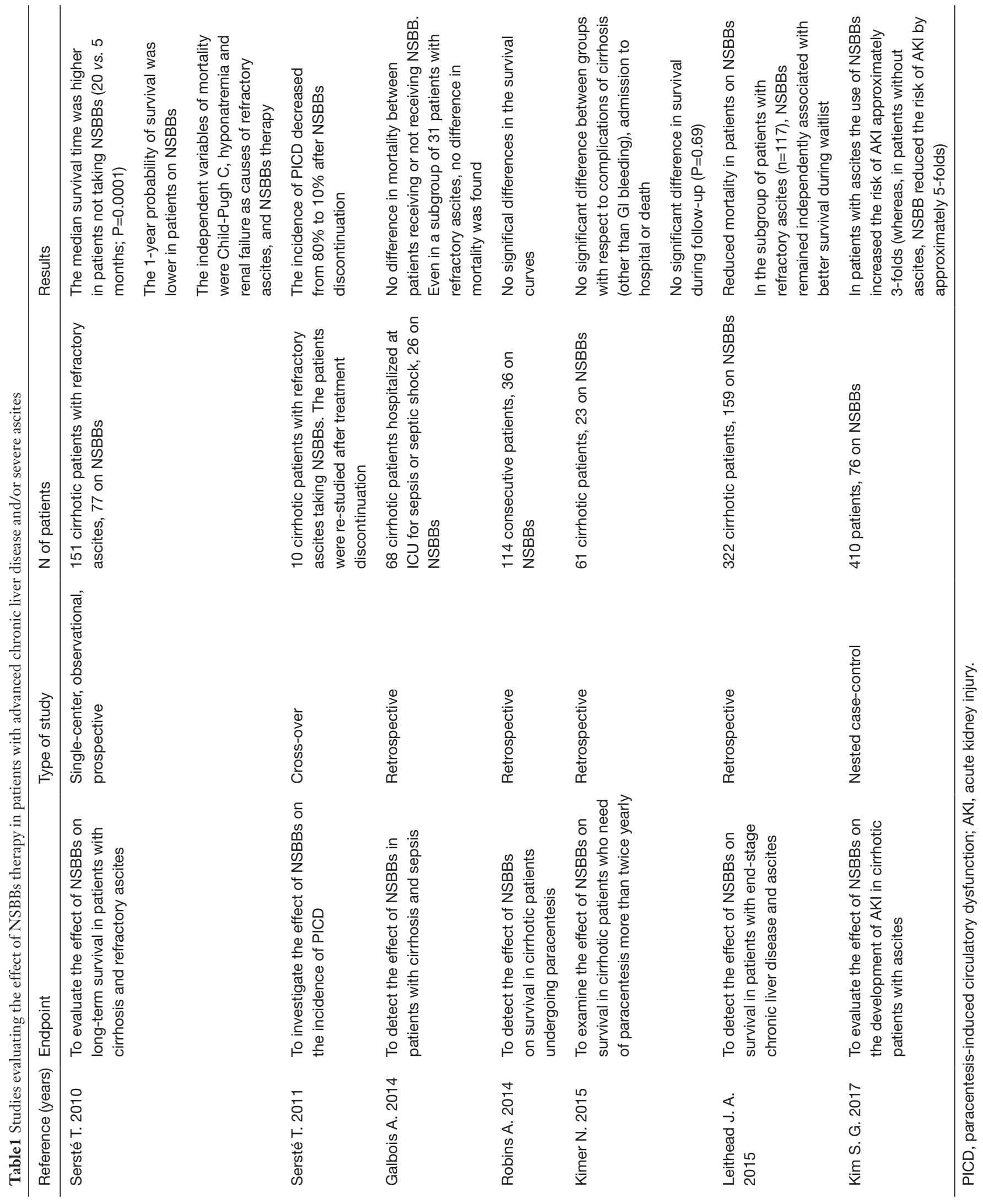


rate of death (adjusted HR $=0.35 ; 95 \%$ CI, 0.14-0.86; $\mathrm{P}=0.022)(8)$.

Based on these findings, clinicians are continuously facing the need to evaluate pros and cons on the use of NSBBs in patients with advanced cirrhosis. In fact, while on the one hand the NSBBs represent a crucial option for primary and secondary prophylaxis of variceal bleeding, on the other hand caution has been suggested as they may reduce the cardiac response to the hyperdynamic circulation, leading to cardiac dysfunction and further deterioration in decompensated cirrhotic patients $(9,10)$.

The Baveno VI international consensus conference held in 2015 recommended that, in patients with cirrhosis and refractory ascites, NSBBs should be used cautiously with close monitoring of blood pressure, serum sodium, and serum creatinine. In particular, NSBBs should be reduced/discontinued if a patient with refractory ascites develops systolic blood pressure $<90 \mathrm{mmHg}$, hyponatremia $<130 \mathrm{mEq} / \mathrm{L}$ or acute kidney injury (AKI). Re-initiation of NSBB should be considered after these abnormal parameters return to baseline values or after resolution of the precipitant; in such case, dose should be re-titrated, starting at the lowest dose. The consensus also underlined the need to reassess the risks/benefits ratio of NSBB periodically in each patient, as contraindications, such as hypotension, may be absent when the therapy is initiated but may eventually appear later (11). The AASLD 2016 clinical guidelines provide similar recommendations, adding the suggestion of avoiding doses of more than $160 \mathrm{mg}$ in the case of propranolol or $80 \mathrm{mg}$ when using nadolol in patients with refractory ascites and spontaneous bacterial peritonitis (SBP) (12).

In patients with advanced cirrhosis, development of AKI represents an important landmark in the disease progression (13). When an organic kidney disease is not the cause of AKI, hyperdynamic circulation and cardiac dysfunction are the main determinants of renal impairment in cirrhotic patients, which may eventually progress to hepatorenal syndrome. Even in presence of a pre-existent chronic kidney disease, hemodynamic deterioration may contribute to further worsening kidney function.

Kim et al. (14) performed a nested case-control study from a cohort of patients listed for liver transplantation, aimed at evaluating the association between NSBBs treatment and the development of AKI. They analyzed 410 patients, cases were patients with AKI (205 patients) and controls were matched patients, based on MELD-Na, age, baseline creatinine and duration of follow-up, who did not develop AKI. The unadjusted proportional hazard regression analysis for predicting the development of AKI, showed that non-Caucasian race, high MELD and MELD$\mathrm{Na}$ scores at baseline, and ascites were associated with an increased risk of AKI. To investigate further the influence of ascites and of the use of NSBBs the patients were divided into 4 subgroups: in patients with ascites the use of NSBB increased the risk of AKI approximately 3 -folds $(\mathrm{HR}=3.31$; 95\% CI, 1.57-6.95; $\mathrm{P}<0.01)$; whereas, in patients without ascites, NSBB reduced the risk of AKI by approximately 5 -fold $(\mathrm{HR}=0.19 ; 95 \% \mathrm{CI}, 0.06-0.60 ; \mathrm{P}<0.01)$. These results need to be considered with interest, as those of other observational studies. On one side, NSBBs seem to favor the development of AKI in patients with cirrhosis and ascites, on the other side NSBBs seem to protect from AKI patients with cirrhosis and without ascites.

These findings seem to support the "window hypothesis" which proposed that NSBBs improve survival in cirrhosis only during a certain "window" of the disease (8): not too early, when the adrenergic system is not yet activated in the initial stages of cirrhosis, and not too late, when a number of circulatory changes occur for an adaptive response to the peripheral vasodilation, effective hypovolemia, and arterial hypotension. At this advanced stage, the effects of NSBBs in reducing blood pressure and cardiac output may result in decreased survival in a subset of patients (12). The study by Kim suggests that even the presence of ascites can be a risk factor for the use of NSBBs. However, as patients with ascites were not excluded from the old trials considering NSBBs for the primary and secondary prevention of variceal bleeding in cirrhotic patients, and shared the same beneficial effects of the therapy with beta-blockers, these results are difficult to be interpreted. Although the authors tried to overcome the limitations of an observational retrospective design by planning a nested case-control study, many problems could still have influenced the results. A relevant limitation of the study is due to the fact that the use of NSBBs could be a marker of more advanced cirrhosis even in patients with ascites, identifying those with more severe portal hypertension, and multivariable analysis has limited power to account for all potential confounders. It is not reported whether patients with ascites, receiving and not receiving NSBBs, were comparable for MELD, MELD-Na and hemodynamic parameters. Moreover, being 
a retrospective study, as also stated by the authors, it was not possible to assess accurately the severity of ascites, and this may represent an important bias in a study in which the conclusions involve the presence or absence of ascites. Another important point to take into account is that, in cirrhotic patients, AKI can have different causes (organic and functional AKI) and these may also affect the influence of multiple factors which may act as confounders. Detailed information about $\mathrm{AKI}$ is also lacking.

In conclusion, prospective RCT would be needed on the use of NSBBs in decompensated cirrhotic patients with ascites however these trials are difficult to organize and will need a large sample size. A case control study could be useful if the two groups were patients matched for MELD, MELD $\mathrm{Na}$, creatinine, mean arterial pressure, with the same type of ascites (refractory, recurrent, severe) taking or not taking NSBBs. The better and more relevant end point should probably be survival.

In the meanwhile, the Baveno recommendations can be utilized in clinical practice to remind that severe hypotension is a well-known contraindication for NSBBs which may suggest dose reduction or even therapy discontinuation.

Last but not least, NSBBs may have several beneficial effects in patients with cirrhosis beyond the reduction in portal hypertension. They reduce markers of intestinal permeability, bacterial translocation and systemic inflammation, and also the risk of $\operatorname{SBP}(15,16)$. The risk to enlarge the indication to stop NSBBs in cirrhotic patients without a real evidence could be as to "throw the baby out with the bath water".

\section{Acknowledgements}

None.

\section{Footnote}

Conflicts of Interest: The authors have no conflicts of interest to declare.

\section{References}

1. Lebrec D, Nouel O, Corbic M, et al. Propranolol-a medical treatment for portal hypertension? Lancet 1980;2:180-2.

2. Moctezuma-Velazquez C, Kalainy S, Abraldes JG. Beta- blockers in patients with advanced liver disease: Has the dust settled? Liver Transpl 2017;23:1058-69.

3. Sersté T, Melot C, Francoz C, et al. Deleterious effects of beta-blockers on survival in patients with cirrhosis and refractory ascites. Hepatology 2010;52:1017-22.

4. Sersté T, Francoz C, Durand F, et al. Beta-blockers cause paracentesis-induced circulatory dysfunction in patients with cirrhosis and refractory ascites: a cross-over study. J Hepatol 2011;55:794-9.

5. Galbois A, Das V, Thabut D, et al. Beta-blockers have no effect on outcomes in patients with cirrhosis and severe infections. Hepatology 2011;53:1412-3.

6. Robins A, Bowden A, Watson W, et al. Beta-blockers in cirrhosis patients with refractory ascites. Hepatology 2014;59:2054-5.

7. Kimer N, Feineis M, Møller S, et al. Beta-blockers in cirrhosis and refractory ascites: a retrospective cohort study and review of the literature. Scand J Gastroenterol 2015;50:129-37.

8. Leithead JA, Rajoriya N, Tehami N, et al. Non-selective $\beta$-blockers are associated with improved survival in patients with ascites listed for liver transplantation. Gut 2015;64:1111-9.

9. Krag A, Wiest R, Albillos A, et al. The window hypothesis: haemodynamic and non-haemodynamic effects of $\beta$-blockers improve survival of patients with cirrhosis during a window in the disease. Gut 2012;61:967-9.

10. Giannelli V, Lattanzi B, Thalheimer U, et al. Beta-blockers in liver cirrhosis. Ann Gastroenterol 2014;27:20-6.

11. de Franchis R, Baveno VI Faculty. Expanding consensus in portal hypertension: Report of the Baveno VI Consensus Workshop: Stratifying risk and individualizing care for portal hypertension. J Hepatol 2015;63:743-52.

12. Garcia-Tsao G, Abraldes JG, Berzigotti A, et al. Portal hypertensive bleeding in cirrhosis: Risk stratification, diagnosis, and management: 2016 practice guidance by the American Association for the study of liver diseases. Hepatology 2017;65:310-35.

13. Angeli P, Gines P, Wong F, et al. Diagnosis and management of acute kidney injury in patients with cirrhosis: revised consensus recommendations of the International Club of Ascites. Gut 2015;64:531-7.

14. Kim SG, Larson JJ, Lee JS, et al. Beneficial and harmful effects of nonselective beta blockade on acute kidney injury in liver transplant candidates. Liver Transpl 2017;23:733-40.

15. Reiberger T, Ferlitsch A, Payer BA, et al. Non-selective 
betablocker therapy decreases intestinal permeability and serum levels of LBP and IL-6 in patients with cirrhosis. J Hepatol 2013;58:911-21.

16. Merli M, Lucidi C, Di Gregorio V, et al. The chronic

doi: 10.21037/amj.2017.08.08

Cite this article as: Lattanzi B, Di Gregorio V, D'Ambrosio

$\mathrm{D}$, Merli M. The use of non-selective beta-blockers in patients with cirrhosis: more doubts than certainties. AME Med J 2017;2:113. use of beta-blockers and proton pump inhibitors may affect the rate of bacterial infections in cirrhosis. Liver Int 2015;35:362-9. 